\title{
Mixing Fingerprints For Generating Virtual Identities
}

\author{
Asem Othman ${ }^{\# 1}$ and Arun Ross $\# 2$ \\ \# Lane Department of Computer Science and Electrical Engineering, \\ West Virginia University, USA \\ 1 asem.othman@mail.wvu.edu \\ 2 arun.ross@mail.wvu.edu
}

\begin{abstract}
This work explores the possibility of mixing two different fingerprints at the image level in order to generate a new fingerprint. To mix two fingerprints, each fingerprint is decomposed into two different components, viz., the continuous and spiral components. After pre-aligning the components of each fingerprint, the continuous component of one fingerprint is combined with the spiral component of the other fingerprint image. Experiments on a subset of the WVU fingerprint dataset show that the proposed approach can be used to generate virtual identities from images of two different fingers pertaining to a single individual or different individuals.
\end{abstract}

\section{INTRODUCTION}

Image level fusion refers to the consolidation of (a) multiple samples of the same biometric trait obtained from different sensors or (b) multiple instances of the same biometric trait obtained using a single sensor, in order to generate a new image [20]. In the context of fingerprints, image-level fusion has been used to combine multiple impressions of the same finger as exemplified in the following scenarios:

Multispectral sensor: Rowe et al. [24] fused multiple images acquired from a multispectral fingerprint scanner into a single high quality fingerprint image.

Small-area sensor: Some sensors capture only a small portion of the fingertip [15]. Therefore, several fingerprint mosaicking techniques [19], [11], [16], [4], [23], [27] have been developed to stitch multiple impressions of the same finger and create a larger fingerprint.

Multi-view sensor: Touchless fingerprint sensors capture multiple views of a finger using several calibrated cameras [18] or a single camera with two planar mirrors [3]. These multiple views are mosaicked together to yield a single nail-to-nail fingerprint.

In this paper, unlike previous work, two fingerprint impressions acquired from two different fingers are fused into a new fingerprint image resulting in a new identity ${ }^{1}$. The mixed image [21] incorporates characteristics from both the original fingerprint images, and can be used directly in the feature extraction and matching stages of an existing biometric system. There are several benefits for mixing fingerprints. For example, the proposed approach could be used to fuse images of the thumb and the index fingers of a single individual,

\footnotetext{
${ }^{1}$ Here, the term "identity" is used to suggest that the mixed fingerprint is unique and possibly different from other fingerprints.
}

or index fingers of two different individuals. Therefore, the concept of mixing fingerprints could be utilized in a multifinger authentication system. Further, fingerprint mixing can be used to generate a large set of virtual identities. These virtual identities can be used to conceal the original identities of subjects or be used for large-scale evaluation of algorithms [2][15]. The mixing process begins by decomposing each fingerprint image into two different components, viz., the continuous and spiral components (see Figure 1). Next, the two components of each fingerprint are aligned to a common coordinate system. Finally, the continuous component of one fingerprint is combined with the spiral component of the other fingerprint. The experimental results confirm that (a) the new fingerprint .representing a new identity, can potentially be used for authentication; (b) the mixed fingerprint is dissimilar from the original fingerprints; and (c) the proposed method can be utilized to generate different-sized databases of virtual identities from a fixed fingerprint dataset. The rest of the

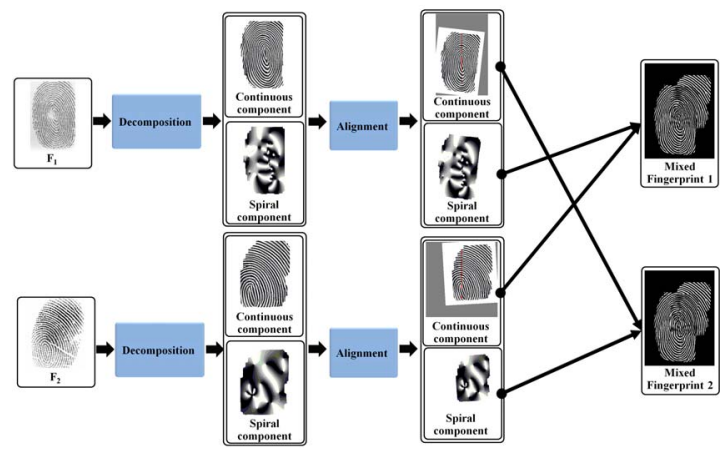

Fig. 1. Proposed approach for mixing fingerprints

paper is organized as follows. Section II presents the proposed approach for mixing fingerprints. Section III reports the experimental results and Section IV concludes the paper.

\section{MIXING FINGERPRINTS: THE PROPOSED APPROACH}

The ridge flow of a fingerprint can be represented as a $2 \mathrm{D}$ Amplitude and Frequency Modulated (AM-FM) signal [14]:

$$
I(x, y)=a(x, y)+b(x, y) \cos (\Psi(x, y))+n(x, y),
$$

where $I(x, y)$ is the intensity of the original image at $(x, y)$, $a(x, y)$ is the intensity offset, $b(x, y)$ is the amplitude, $\Psi(x, y)$ 
is the phase and $n(x, y)$ is the noise. Based on the Helmholtz Decomposition Theorem [7], the phase can be uniquely decomposed into the continuous phase and the spiral phase, $\Psi(x, y)=\psi_{c}(x, y)+\psi_{s}(x, y)$. As shown in Figure 2, the cosine of the continuous phase, i.e., the continuous component $\cos \left(\psi_{c}(x, y)\right)$, defines the local ridge orientation, and the cosine of the spiral phase, i.e., the spiral component $\cos \left(\psi_{s}(x, y)\right)$, characterizes the minutiae locations. Let $\phi(x, y)$ denote the

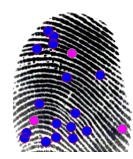

(a)

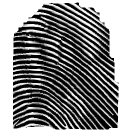

(b)

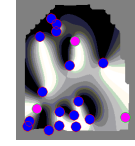

(c)
Fig. 2. Decomposing a fingerprint. (a) A fingerprint image. (b) Continuous component, $\cos \left(\psi_{c}(x, y)\right)$. (c) Spiral component, $\cos \left(\psi_{s}(x, y)\right)$. The blue and pink dots represent ridge endings and ridge bifurcations, respectively.

spiral phase of a local region in a fingerprint. Assume that the function $\phi(x, y)$ monotonically changes from 0 to $2 \pi$ around a particular point, $\left(x_{n}, y_{n}\right)$, and has a characteristic jump from 0 to $2 \pi$ at the point $\left(x_{n}, y_{n}\right)$. This forms a residue at $\left(x_{n}, y_{n}\right)$ with an associated polarity, $p_{n} \in\{-1,1\}$. A residue with positive (negative) polarity is referred to as a positive (negative) spiral.

$$
\phi(x, y)=p_{n} \tan ^{-1}\left(\left(x-x_{n}\right) /\left(y-y_{n}\right)\right) .
$$

Appending this function to the continuous phase will cause a phase jump at $\left(x_{n}, y_{n}\right)$ resulting in a minutia. In Figure 3 , a local ridge pattern is generated based on the continuous phase function $2 \pi f y$, with $f=4$. Depending upon the polarity value $(+1$ or -1$)$, a minutia is generated on the ridge pattern. The relation between the polarity, $p_{n}$, and the occurrence of ridge ending or bifurcation is dependent on the gradient direction of the cosine of the continuous phase. Hence, the spiral phase allows for an abrupt change in the local fringe density by either inserting or deleting a ridge based on the polarity and the appending location within the continuous phase. If the simple

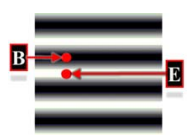

(a)
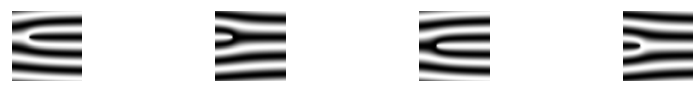
(b) $p_{n}=+1$
(c) $p_{n}=-1$
(d) $p_{n}=+1$
(e) $p_{n}=-1$

Fig. 3. Generating minutia in a fringe pattern. (a) Gray scale image of continuous phase given by $\cos (2 \pi f y)$. (b) and(c) Appending a minutia at "B". (d) and (e) Appending a minutia at "E".

function in (2) is replaced by a sum of such functions, the spiral phase, $\psi_{s}(x, y)$, will correspond to a set of minutiae:

$$
\psi_{s}(x, y)=\sum_{n=1}^{N} p_{n} \tan ^{-1}\left(\left(x-x_{n}\right) /\left(y-y_{n}\right)\right)
$$

where $x_{n}$ and $y_{n}$ denote the coordinates of the $n^{\text {th }}$ minutia, and $N$ denotes the total number of minutiae. Moreover, the type of a minutia (ending or bifurcation) is determined by its polarity $p_{n} \in\{-1,1\}$. Thus, based on this $2 \mathrm{D}$ AM-FM representation, the fingerprint's oriented patterns can be uniquely decomposed into (a) a small number of topologically distinct discontinuities, i.e., the spiral phase, and (b) a well defined smooth flow field, i.e., the continuous phase.

\section{A. Fingerprint Decomposition}

Since ridges and minutiae can be completely determined by the phase, we are only interested in $\Psi(x, y)$. The other three parameters in Equation (1) contribute to the realistic textural appearance of the fingerprint. Before fingerprint decomposition, the phase $\Psi(x, y)$ must be reliably estimated; this is termed as demodulation.

1) Vortex demodulation: The objective of vortex demodulation [13] is to extract the amplitude $b(x, y)$ and phase $\Psi(x, y)$ of the fingerprint pattern. First, the DC term $a(x, y)$ has to be removed since the failure to remove this offset correctly may introduce significant errors in the demodulated amplitude and phase [13]. To facilitate this, a normalized fingerprint image, $f(x, y)$, containing the enhanced ridge pattern of the fingerprint (generated by the VeriFinger $\mathrm{SDK}^{2}$ ) is used. From Equation (1), $f(x, y)=I(x, y)-a(x, y) \simeq b(x, y) \cos (\Psi(x, y))$. The vortex demodulation operator $\mathbf{V}$ takes the normalized image $f(x, y)$ and applies a spiral phase Fourier multiplier $\exp [i \Phi(u, v)]$ :

$$
\begin{aligned}
& \mathbf{V}\{f(x, y)\}=F^{-1}\{\exp [i \Phi(u, v)] \cdot F\{b(x, y) \cdot \cos [\Psi(x, y)]\}\} \\
& \cong-i \exp [i \beta(x, y)] \cdot b(x, y) \cdot \sin [\Psi(x, y)]
\end{aligned}
$$

where, $F$ is the Fourier transform, $F^{-1}$ is the inverse Fourier transform and $\exp [i \Phi(u, v)]$ is a 2-D signum function [13] defined as a pure spiral phase function in the spatial frequency space $(u, v)$ :

$$
\exp [i \Phi(u, v)]=\frac{u+i v}{\sqrt{u^{2}+v^{2}}} .
$$

Note that in Equation (4) there is a new parameter, $\beta(x, y)$, representing the perpendicular direction of the ridges. In Equation (6), this directional map is used to isolate the desired magnitude and phase from Equation (4), i.e.,

$$
-\exp [-i \beta(x, y)] . \mathbf{V}\{f(x, y)\}=i b(x, y) \cdot \sin [\Psi(x, y)] .
$$

Then, Equation (6) can be combined with the normalized image, $f(x, y)$, to obtain the magnitude $b(x, y)$ and the raw phase map $\Psi(x, y)$ as follows:

$$
-\exp [-i \beta(x, y)] . \mathbf{V}\{f(x, y)\}+f(x, y)=b(x, y) \cdot \exp (i \Psi(x, y)) .
$$

Therefore, determining $\beta(x, y)$ is essential for obtaining the amplitude and phase functions, $b(x, y)$ and $\Psi(x, y)$, respectively. The direction map $\beta(x, y)$ can be derived from the orientation image of the fingerprint by a process called unwrapping. A sophisticated unwrapping technique using the topological

\footnotetext{
${ }^{2}$ http://www.neurotechnology.com
} 
properties of the ridge flow fields is necessary to account for direction singularities such as cores and deltas [14] [6].

2) Direction Map $\beta(x, y)$ : Direction is uniquely defined in the range $0^{\circ}$ to $360^{\circ}$ (modulo $2 \pi$ ). In contrast, fingerprint ridge orientation is indistinguishable from that of a $180^{\circ}$ rotated ridge (modulo $\pi$ ). Therefore, the fingerprint's orientation map, denoted by $\theta(x, y)$, should be unwrapped to a direction map, $\beta(x, y)$ [14]. Phase unwrapping is a technique used to address a $2 \pi$ phase jump in the orientation map. The unwrapping process adds or subtracts an offset of $2 \pi$ to successive pixels whenever a phase jump is detected [7]. This process proceeds by starting at any pixel within the orientation image and using the local orientation information to traverse the image pixel-by-pixel, and assigning a direction (i.e., the traversed direction) to each pixel with the condition that there are no discontinuities of $2 \pi$ between neighboring pixels. However, the presence of flow singularities means that there will be pixels in the orientation image with a discontinuity of $\pm 2 \pi$ in the traversed direction and, therefore, the above unwrapping technique will fail. In fingerprint images, such flow singularities arise from the presence of singular points such as core and delta. Figure 4(a) illustrates that estimating the direction of ridges in the vicinity of a core point by starting at any point within the highlighted rectangle and arbitrarily assigning one of two possible directions, can result in an inconsistency in the estimated directions inside the dashed circle. This inconsistency in the estimated direction map can be avoided by using a branch cut [7]. The branch cut is a line or a curve used to isolate the flow singularity and which cannot be crossed by the paths of the unwrapping process. Consequently, branch cut prevents the creation of $2 \pi$ discontinuities and restores the path independence of the unwrapping process. As shown in Figure 4(b), tracing a line down from the core point and using this line as a barrier resolves the inconsistency near the core point (i.e, inside the dashed circle) by selecting two different directions in each side of the branch cut within the same region (i.e, inside the highlighted rectangle). The adopted strategy,

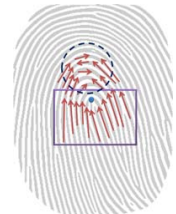

(a)

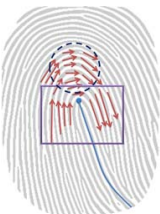

(b)
Fig. 4. A portion of the estimated direction map (a) before assigning a branch cut and (b) after assigning a branch cut [9].

based on the techniques described in [14] [9] [6] to estimate the direction map $\beta(x, y)$, is summarized in the following three steps.

1. The orientation image $\theta(x, y)$ of the normalized fingerprint $f(x, y)$ is determined via the least mean-square method [10]. Then the Poincare [15] index is used to locate the singular points, if any.

2. In case there are singular points, an algorithm is applied to extract the branch cuts along suitable paths such as ridge contours, as shown in Figure 4(b), to resolve the inevitable direction ambiguities near those singularities. The branch cuts are extracted by tracing the contours of ridges (rather than the orientation field) in the skeleton images. The algorithm starts from each singular point in a skeleton image until the trace reaches the border of the segmented foreground region of the fingerprint or when it encounters another singular point. To generate the skeleton images, first, a set of smoothed orientation maps are generated by applying a Gaussian smoothing operation at different smoothing scales $(\sigma \in\{1,2,3,5,10,15,20,32,50,64\})$ on $\theta(x, y)$. Next, a set of Gabor filters, tuned to the smoothed orientation maps [10], is convolved with the normalized image $f(x, y)$. Then, a local adaptive thresholding and thinning algorithm [25] is applied to the directionally filtered images producing 10 skeleton images. Thus, there are at least 10 branch cuts and the shortest one, associated with each singular point, is selected. Figure 5 shows the final extracted branch cuts from all singular points.

3. The phase unwrapping algorithm [8] [7] starts from any arbitrary pixel in the orientation map $\theta(x, y)$ and visits the other pixels, which are unwrapped in the same manner as in images without singularity, with the exception here that the branch cuts cannot be crossed. Then, each branch cut is visited individually and its pixels are traced and unwrapped. Finally,

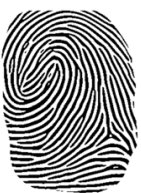

(a) Normalized Fingerprint

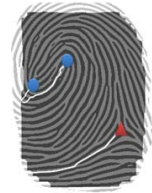

(b) Branch Cuts
Fig. 5. Example of a fingerprint with three singular points (2 cores and 1 delta). (a) The normalized fingerprint. (b) The extracted branch cuts obtained by tracing the ridges instead of the orientation field.

the direction map $\beta(x, y)$ is determined from the unwrapped $\theta(x, y)$ by adding $\pi / 2$ which allows for the determination of the amplitude $b(x, y)$ and phase $\Psi(x, y)$ modulations of fingerprint image from Equation (7).

3) Helmholtz Decomposition: The Helmholtz Decomposition Theorem [7] is used to decompose the determined phase $\Psi(x, y)$ of a fingerprint image into two phases. The first phase, $\psi_{c}$ is a continuous one, which can be unwrapped, and the second is a spiral phase, $\psi_{s}$, which cannot be unwrapped but can be defined as a phase that exhibits spiral behavior at a set of discrete points in the image. The Bone's residue detector [1] [7] is first used to determine the spiral phase $\psi_{s}(x, y)$ from the demodulated phase $\Psi(x, y)$. Next the continuous phase, is computed as $\psi_{c}(x, y)=\Psi(x, y)-\psi_{s}(x, y)$. Finally, the extracted branch cuts from the previous step are used again to unwrap the continuous phase.

\section{B. Fingerprint Pre-alignment}

To mix two different fingerprints after decomposing each fingerprint into its continuous component $\cos \left(\psi_{c}(x, y)\right)$ and 
spiral component $\cos \left(\psi_{s}(x, y)\right)$, the fingerprint themselves should be appropriately aligned. Previous research has shown that two fingerprints can be best aligned using their minutiae correspondences. However, it is difficult to ensure the existence of such correspondences between two fingerprints acquired from different fingers. In this paper, the components are pre-aligned to a common coordinate system prior to the mixing step by utilizing a reference point and an alignment line. The reference point is used to center the components. The alignment line is used to find a rotation angle about the reference point. This angle rotates the alignment line to make it vertical. The two phase components of each fingerprint are rotated by the same angle.

1) Locating a reference point: The reference point used in this work is the northern most core point of extracted singularities. For plain arch fingerprints or partial fingerprint images, Novikov et al.'s technique [17] [22], based on the Hough transform, is used to detect the reference point.

2) Finding the alignment line: The first step in finding the alignment line is to extract high curvature points from the skeleton of the fingerprint image's continuous component. Next, horizontal distances between the reference point and all high curvature points are calculated. Then, based on these distances, an adaptive threshold is applied to select and cluster points near the reference point. Finally, a line is fitted through the selected points to generate the alignment line.

\section{Mixing Fingerprints}

Let $F_{1}$ and $F_{2}$ be two different fingerprint images from different fingers, and let $\psi_{c i}(x, y)$ and $\psi_{s i}(x, y)$ be the prealigned continuous and spiral phases, $i=1,2$. As shown in Figure 1, there are two different mixed fingerprint image that can be generated, $M F_{1}$ and $M F_{2}$ :

$$
\begin{aligned}
& M F_{1}=\cos \left(\psi_{c 2}+\psi_{s 1}\right), \\
& M F_{2}=\cos \left(\psi_{c 1}+\psi_{s 2}\right) .
\end{aligned}
$$

The continuous phase of $F_{2}\left(F_{1}\right)$ is combined with the spiral phase of $F_{1}\left(F_{2}\right)$ which generates a new fused fingerprint image $M F_{1}\left(M F_{2}\right)$.

\section{Compatibility Measure}

Variations in the orientations and frequencies of ridges between fingerprint images can result in visually unrealistic mixed fingerprint images, as shown in Figure 6. This issue can be mitigated if the two fingerprints to be mixed are carefully chosen using a compatibility measure. In this paper, the compatibility between fingerprints is computed using nonminutiae features, viz., orientation fields and frequency maps of fingerprint ridges. The orientation and frequency images are computed from the pre-aligned continuous component of a fingerprint using the technique described in [10]. Then, Yager and Amin's [26] approach is used to compute the compatibility measure. To compute the compatibility between two fingerprint images, their orientation fields and frequency maps are first estimated (see below). Then, the compatibility measure $C$ between them is computed as the weighted sum
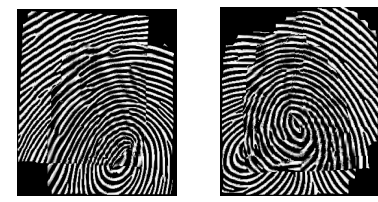

Fig. 6. Examples of mixed fingerprints that look unrealistic.

of the normalized orientations and frequency differences, $O D$ and $F D$, respectively:

$$
C=1-(\alpha . O D+\gamma \cdot F D),
$$

where $\alpha$ and $\gamma$ are weights that are determined empirically. Figure 7 shows examples of mixed fingerprints after utilizing the compatibility measure ${ }^{3}$ to select the fingerprints pairs, $\left(F_{1}\right.$, $F_{2}$ ).

1) Orientation Fields Difference $(O D)$ : The difference in orientation fields between $F_{1}$ and $F_{2}$ is computed as

$$
O D=\left(\frac{1}{S}\right) \sum_{(x, y) \in S} d\left(\theta_{1}(x, y), \theta_{2}(x, y)\right),
$$

where $S$ is a set of coordinates within the overlapped area of the aligned continuous components of two different fingerprints, and $\theta_{1}$ and $\theta_{2}$ represent the orientation fields of the two fingerprints. If orientations are restricted to the range $[-\pi / 2, \pi / 2]$, the operator $d($.$) is written as$

$$
d(\alpha, \gamma)= \begin{cases}\pi-(\alpha-\gamma), & \text { if } \frac{\pi}{2}<\alpha-\gamma \\ |\alpha-\gamma|, & \text { if }-\frac{\pi}{2}<\alpha-\gamma<\frac{\pi}{2} \\ \pi+(\alpha-\gamma), & \text { if } \alpha-\gamma \leq-\frac{\pi}{2} .\end{cases}
$$

2) Frequency Maps Difference $(F D)$ : Local ridge frequencies are the inverse of the average distance between ridges in the local area in a direction perpendicular to the local orientation. Hong et al.'s approach [10] is used to find the local ridge frequencies of the continuous component of a fingerprint image. The difference function is computed as

$$
F D=\left(\frac{1}{S}\right) \sum_{(x, y) \in S} \mid \text { Freq }_{1}(x, y)-\text { Freq }_{2}(x, y) \mid,
$$

where $S$ is a set of coordinates within the overlapped area, and $F_{r e q}$ and Freq $_{2}$ represent the frequency maps of the two fingerprints $F_{1}$ and $F_{2}$, respectively.

\section{EXPERIMENTS AND DISCUSSION}

The performance of the proposed approach to generate virtual identities was tested using the fingerprint dataset from the West Virginia University (WVU) multimodal biometric database [5]. A subset of 300 images corresponding to 150 fingers (two impressions per finger) was used. For each finger

\footnotetext{
${ }^{3}$ Perfect compatibility $(C=1)$ is likely to occur when the two prints to be mixed are from the same finger - a scenario that is not applicable in the proposed application. On the other hand, two fingerprints having significantly different ridge structures are unlikely to be compatible $(C=0)$ and will generate an unrealistic looking fingerprint. Between these two extremes, lies a range of possible compatible values that is acceptable. However, determining this range automatically may be difficult.
} 
in the WVU dataset, one impression was used as the probe image and the other was added to the gallery resulting in a probe set $P$ and gallery set $G$ each containing 150 fingerprints. In the following experiments, the VeriFinger SDK was used to generate the normalized fingerprint images and the matching scores. Also, an open source Matlab implementation [12] based on Hong et al.'s approach [10] was used to compute the orientation and frequency maps of the fingerprints. In order to establish the baseline performance, the images in $P$ were matched against those in $G$. This resulted in a rank-1 accuracy of $100 \%$ and an Equal Error Rate (EER) of 0\%. The experiments were designed in order to address the following questions:

1. What impact does mixing fingerprints have on the matching performance, i.e., can two mixed impressions pertaining to the same new identity be successfully matched?

2. Are the original fingerprints and the mixed fingerprint correlated? It is essential to assure that the proposed approach generates a new fingerprint that is dissimilar from the original fingerprints.

3. How many virtual identities can be generated from a fixed fingerprint dataset with an acceptable recognition rate?

Experiment 1: In this experiment, the performance of generating new identities by mixing random pairs of fingers is reported. Pairs of fingerprints in $P$ were randomly paired and mixed resulting in a new probe set $M F_{1}^{P}$ consisting of 75 fingerprints. The corresponding pairs of fingerprints in $G$ were also mixed resulting in a new gallery set $M F_{1}^{G}$ consisting of 75 impressions. Since, mixing is an asymmetric process (Equation (8)), another probe set $M F_{2}^{P}$ and gallery set $M F_{2}^{G}$ were also generated. Matching $M F_{1}^{P}$ against $M F_{1}^{G}$ and $M F_{2}^{P}$ against $M F_{2}^{G}$ resulted in a rank-1 accuracy of $\sim 68 \%$ and an EER of $\sim 15 \%$. The low identification rate is due to the random pairing of fingers which lead to visually unrealistic fingerprint images (see Figure 6).

Experiment 2: The purpose of this experiment is to enhance the identification rate of Experiment 1 by mixing fingers based on the compatibility measure. Therefore, the compatibility measure between different pairs of fingerprints in $P$ were computed using Equation (9) with $\alpha=0.7$ and $\gamma=0.3$. The finger pairs to be mixed were selected based on these values. Pairs were selected and mixed in decreasing order of their compatibility values resulting in probe sets $M F_{1}^{P}$ and $M F_{2}^{P}$, and gallery sets $M F_{1}^{G}$ and $M F_{2}^{G}$. Figure 7 shows examples of the mixed fingerprints from the WVU dataset. Matching $M F_{1}^{P}$ against $M F_{1}^{G}$ and $M F_{2}^{P}$ against $M F_{2}^{G}$ resulted in a rank1 accuracy of $\sim 86 \%$ and an EER of $\sim 4 \%$. The reasonably high recognition rates (compared to Experiment 1) indicate the possibility of mixing fingerprints based on their compatibility measure. As shown in Figures 6 and 7, the compatibility measure assists the mixing approach in generating visually appealing mixed fingerprints with less false minutia in the overlapping area. Currently, ways to further improve the rank1 accuracy of mixed fingerprints is being examined.

Experiment 3: It is essential to assure that the new identities are dissimilar from the original fingers. Therefore, in this

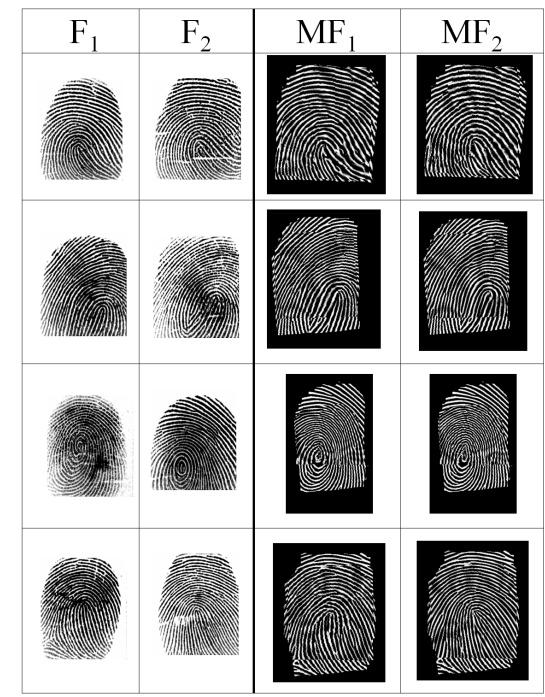

Fig. 7. Examples of mixing fingerprint pairs from the WVU dataset.

experiment, $M F_{1}^{P}$ and $M F_{2}^{P}$, generated in Experiment 2, are matched against $F_{1}^{P}$ and $F_{2}^{P}$ in $P$ (as in Experiment $2, F_{1}^{P}$ and $F_{2}^{P}$ are paired and mixed based on the compatibility measures, and $M F_{1}^{P}$ and $M F_{2}^{P}$ are the resulting mixed fingerprints).

a. Matching $M F_{1}^{P^{2}}\left(M F_{2}^{P}\right)$ against $F_{1}^{P}\left(F_{2}^{P}\right)$ resulted in rank-1 accuracy of $\sim 52 \%$ and EER of $\sim 30 \%$.

b. Matching $M F_{1}^{P}\left(M F_{2}^{P}\right)$ against $F_{2}^{P}\left(F_{1}^{P}\right)$ resulted in rank-1 accuracy of $\sim 38 \%$ and EER of $\sim 43 \%$.

The poor matching performance indicates that the original fingerprints are different from newly generated mixed fingerprints. In other words, the original identity cannot be easily deduced from the mixed image and the new mixed fingerprint may be viewed as a cancelable fingerprint [21]. However, in matching scenario "a", the reduction in the dissimilarity between original and mixed fingerprints is because $M F_{1}^{P}$ $\left(M F_{2}^{P}\right)$ and $F_{1}^{P}\left(F_{2}^{P}\right)$ have the same minutia locations as shown in Figure 1 and Equation (8). This commonality of minutia locations leads to high similarity scores between original and virtual identities. Ridge features, e.g., ridge length and ridge curvature, can be used along with conventional minutia features to address the commonality of minutia locations between $M F_{1}^{P}\left(M F_{2}^{P}\right)$ and $F_{1}^{P}\left(F_{2}^{P}\right)$.

Experiment 4: In this experiment, the possibility of utilizing the proposed approach to mix the prints from the two fingers of a subject to create a single new fingerprint is investigated. The new identity is a result of fusing images of the thumb and the index fingers of a single individual. For this experiment, the data corresponding to both the left thumb and left index finger of 150 subjects from the WVU database were used. There were two impressions available for each finger. Each left thumb impression was mixed with the corresponding left index finger resulting in two mixed fingerprint impressions for each subject. One of these mixed impressions was used as a probe and the other was added to the gallery set. The obtained rank-1 accuracy was $\sim 81 \%$ and the EER was $\sim$ 
9\% suggesting the possibility of designing a new multi-finger authentication scheme for access control. Here, only the mixed impression needs to be stored in the database (as opposed to images of individual fingers).

Experiment 5: Mixing fingerprints generates new fused fingerprints, i.e., new identities. Therefore, in this experiment, we investigated the possibility of generating different-sized databases of virtual identities. Mixing all possible pairs from the probe set $(P)$ will result in $\left(\begin{array}{c}150 \\ 2\end{array}\right)=11,175$ different virtual identities pairs. In this experiment, fingerprints pairs in the probe set are sorted based on the compatibility metric values. Then, the $N$ fingerprint pairs with highest compatibility values in $P$ were mixed and so were their corresponding impressions in the gallery set $(G)$. Table I reports the rank-1, rank-5 accuracies and the EERs of the virtual identity datasets created with different values of $N$. These results confirm the possibility of generating virtual identities by mixing fingerprints; however, there is a trade-off between database size and the identification accuracy ${ }^{4}$. This trade-off is because mixing several pairs from the same probe set $P$ can lead to the generation of several identities sharing a common fingerprint $\left(F_{1}\right)$. Assume two fingerprint pairs $\left(F_{a}, F_{b}\right)$ and $\left(F_{a}, F_{c}\right)$ where $F_{b} \neq F_{c}$. Combining the spiral component, $\cos \left(\psi_{s}\right)$, of the common fingerprint $\left(F_{a}\right)$, with the continuous components, $\cos \left(\psi_{c}\right)$, of $F_{b}$ and $F_{c}$ generates two mixed fingerprints $M F_{a b}$ and $M F_{a c}$, respectively. $M F_{a b}$ and $M F_{a c}$ are likely to share some common minutiae locations. This leads to high impostor matching scores between two different virtual identities, consequently resulting in high false acceptance rate and low identification accuracy.

TABLE I

THE RANK-1, -5 ACCURACIES AND EER OF THE VIRTUAL IDENTITY DATABASES

\begin{tabular}{|c|c|c|c|}
\hline $\begin{array}{c}\text { Size of virtual identity } \\
\text { database }(N)\end{array}$ & Rank-1 (\%) & Rank-5 (\%) & EER (\%) \\
\hline 50 & 88 & 95 & 4 \\
\hline 100 & 85 & 97 & 5 \\
\hline 200 & 84 & 95 & 5 \\
\hline 800 & 68 & 82 & 8 \\
\hline 1000 & 56 & 81 & 10 \\
\hline
\end{tabular}

\section{CONCLUSIONS}

In this work, the possibility of generating a new identity by mixing two distinct fingerprints was explored. To mix two fingerprints, each fingerprint is decomposed into two components, viz., the continuous and spiral components. After aligning the components of each fingerprint, the continuous component of one fingerprint is combined with the spiral component of the other fingerprint image. Experiments on the WVU fingerprint dataset show that (a) the mixed fingerprint representing a new identity can potentially be used for authentication, (b) the mixed fingerprint is dissimilar from the original fingerprints, and (c) the proposed method can be utilized to generate a database of virtual identities from a fixed

\footnotetext{
${ }^{4}$ Generating and matching all the 11,175 virtual identities resulted in an EER of $17 \%$
}

fingerprint dataset. Further work is required to enhance the performance due to mixed fingerprints by exploring alternate algorithms for selecting and mixing the different pairs.

\section{ACKNOWLEDGMENTS}

This project was funded by US NSF CAREER Grant No. IIS 0642554. Thanks to Aglika Gyaourova for her valuable comments.

\section{REFERENCES}

[1] D. Bone. Fourier fringe analysis: the two-dimensional phase unwrapping problem. Applied Optics, 30(25):3627-3632, 1991.

[2] R. Cappelli. Sfinge: Synthetic fingerprint generator. In MSBT Workshop, pages $147-154,2004$.

[3] H. Choi, K. Choi, and J. Kim. Mosaicing touchless and mirror-reflected fingerprint images. TIFS, 5(1):52-61, March 2010.

[4] K. Choi, H. Choi, and J. Kim. Fingerprint mosaicking by rolling and sliding. In AVBPA, pages 260-269, 2005.

[5] S. Crihalmeanu, A. Ross, S. Schuckers, and L. Hornak. A protocol for multibiometric data acquisition, storage and dissemination. Technical report, LCSEE, WVU, 2007.

[6] J. Feng and A. K. Jain. Fingerprint reconstruction: From minutiae to phase. PAMI, 33(2):209 -223, Feb. 2011.

[7] D. Ghiglia and M. Pritt. Two-dimensional phase unwrapping: theory, algorithms, and software. Wiley New York, 1998.

[8] R. Goldstein, H. Zebker, and C. Werner. Satellite radar interferometryTwo-dimensional phase unwrapping. Radio Science, 23(4):713-720, 1988.

[9] B. Hastings. An integrated representation of fingerprint patterns. In CSSE, June 2008

[10] L. Hong, Y. Wan, and A. Jain. Fingerprint image enhancement: algorithm and performance evaluation. PAMI, 20(8):777 -789, aug 1998

[11] A. Jain and A. Ross. Fingerprint mosaicking. In ICASSP, volume 4, pages IV-4064 -IV-4067, 2002.

[12] P. D. Kovesi. MATLAB and Octave functions for computer vision and image processing. CET, The University of Western Australia. Available at: <http://www.csse.uwa.edu.au/ pk/research/matlabfns/ $>$.

[13] K. G. Larkin, D. J. Bone, and M. A. Oldfield. Natural demodulation of two-dimensional fringe patterns. I. General background of the spiral phase quadrature transform. J. Opt. Soc. Am. A, 18(8):1862-1870, 2001.

[14] K. G. Larkin and P. A. Fletcher. A coherent framework for fingerprint analysis: are fingerprints holograms? Opt. Express, 15(14):8667-8677, 2007.

[15] D. Maltoni, D. Maio, A. Jain, and S. Prabhakar. Handbook of fingerprint recognition. Springer, 2009.

[16] Y. Moon, H. Yeung, K. Chan, and S. Chan. Template synthesis and image mosaicking for fingerprint registration: an experimental study. In ICASSP, volume 5, 2004

[17] S. O. Novikov and V. S. Kot. Singular feature detection and classification of fingerprints using hough transform. In DIPCG Workshop, volume 3346, pages 259-269, 1998.

[18] G. Parziale, E. Diaz-Santana, and R. Hauke. The surround imager $\mathrm{tm}$ : A multi-camera touchless device to acquire $3 \mathrm{D}$ rolled-equivalent fingerprints. In Advances in Biometrics, volume 3832, pages 244-250. Springer, 2005.

[19] N. Ratha, J. Connell, and R. Bolle. Image mosaicing for rolled fingerprint construction. In ICPR, volume 2, 1998.

[20] A. Ross, K. Nandakumar, and A. Jain. Handbook of Multibiometrics. Springer, 2006.

[21] A. Ross and A. Othman. Mixing fingerprints for template security and privacy. In EUSIPCO, August 2011.

[22] A. Ross, J. Shah, and A. Jain. From template to image: reconstructing fingerprints from minutiae points. PAMI, 29(4):544-560, 2007.

[23] A. Ross, S. Shah, and J. Shah. Image versus feature mosaicing: a case study in fingerprints. In SPIE BTHI, pages 620208-1 - 620208-12, 2006.

[24] R. Rowe, K. Nixon, and P. Butler. Multispectral fingerprint image acquisition. Advances in Biometrics, pages 3-23, 2008.

[25] R. Thai. Fingerprint image enhancement and minutiae extraction. $\mathrm{PhD}$ thesis, CSSE, The University of Western Australia, 2003.

[26] N. Yager and A. Amin. Fingerprint alignment using a two stage optimization. PRL, 27(5):317-324, 2006.

[27] Y. Zhang, J. Yang, and H. Wu. A hybrid swipe fingerprint mosaicing scheme. In AVBPA, pages 131-140, 2005. 